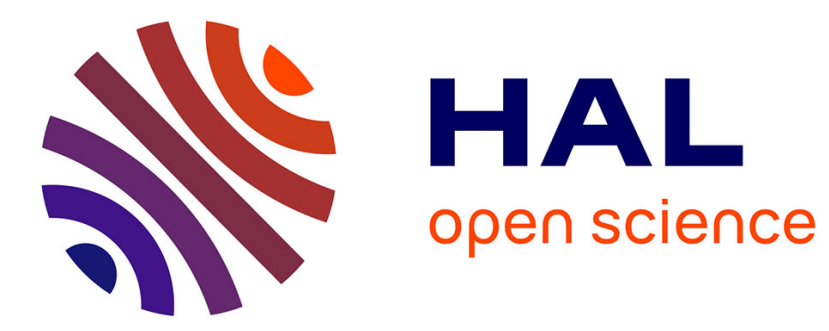

\title{
Tracking control for a class of measure differential inclusions
}

\author{
Nathan van de Wouw, Remco Leine
}

\section{To cite this version:}

Nathan van de Wouw, Remco Leine. Tracking control for a class of measure differential inclusions. 47th IEEE Conference on Decision and Control, Dec 2008, Cancun, Mexico. pp.2526-2532, 10.1109/CDC.2008.4738683 . hal-01309515

\section{HAL Id: hal-01309515 https://hal.science/hal-01309515}

Submitted on 29 Apr 2016

HAL is a multi-disciplinary open access archive for the deposit and dissemination of scientific research documents, whether they are published or not. The documents may come from teaching and research institutions in France or abroad, or from public or private research centers.
L'archive ouverte pluridisciplinaire HAL, est destinée au dépôt et à la diffusion de documents scientifiques de niveau recherche, publiés ou non, émanant des établissements d'enseignement et de recherche français ou étrangers, des laboratoires publics ou privés. 


\title{
Tracking Control for a Class of Measure Differential Inclusions
}

\author{
Nathan van de Wouw, Remco I. Leine
}

\begin{abstract}
In this paper, we study the tracking problem for Lur'e-type measure differential inclusions. The framework of measure differential inclusions allows us to describe systems with discontinuities in the state evolution, such as mechanical systems with unilateral constraints. As a stepping stone, we present results on the stability of time-varying solutions of such systems in the scope of the convergence property. Next, this property is exploited to provide a solution to the tracking problem. The results are illustrated by application to a mechanical motion system with a unilateral velocity constraint.
\end{abstract}

\section{INTRODUCTION}

In this paper, we study the tracking problem for Lur'etype measure differential inclusions. Measure differential inclusions can be used to describe systems which expose discontinuities in the state and/or vector field [1]-[4]. The differential measure of the state vector does not only consist of a part with a density with respect to the Lebesgue measure (i.e. the time-derivative of the state vector), but is also allowed to contain an atomic part. The dynamics of the system is described by an inclusion of the differential measure of the state to a state-dependent set (similar to the concept of differential inclusions). Consequently, the measure differential inclusion concept describes the continuous dynamics as well as the impulsive dynamics with a single statement in terms of an inclusion and is able to describe accumulation phenomena. An advantage of this framework over other frameworks, such as the hybrid systems formalism [5], [6], is the fact that physical interaction laws, such as friction and impact in mechanics or diode characteristics in electronics, can be formulated as set-valued force laws and be seamlessly incorporated in the formulation, see e.g. [7], [8].

Stability properties of measure differential inclusions are essential in the control of such systems. In [8], results on the stability of stationary sets of measure differential inclusions (with a special focus on mechanical systems with unilateral constraints) are presented. In [9], stability properties of an equilibrium of measure differential inclusions of Lur'e-type are studied. The nonlinearities in the feedback loop are required to exhibit monotonicity properties and, if additionally passivity conditions on the linear part of the system are assured, then stability of the equilibrium can be guaranteed. Note that this work studies the stability of stationary solutions. However, many control problems, such as tracking control, output regulation, synchronisation and observer design require the stability analysis of time-varying solutions. The research on the stability and stabilisation of time-varying solutions of non-smooth systems (especially with state jumps) is still in its infancy and the current paper should be placed in this context. It should be noted that the tracking control of measure differential inclusions has received very little attention in literature, see [10]-[12] for works focusing on mechanical systems with unilateral constraints.

In order to study the stability of certain time-varying solutions we consider the framework of convergence. A system, which is excited by an input, is called convergent if it has a unique solution that is bounded on the whole time axis and this solution is globally asymptotically stable. Obviously, if such a solution does exist, then all other solutions converge to this solution, regardless of their initial conditions, and can be considered as a steady-state solution [13]-[15]. The property of convergence can be beneficial in the context of control. In many control problems, such as the tracking problem, it is required that controllers are designed in such a way that all solutions of the corresponding closed-loop system "forget" their initial conditions. Actually, one of the main tasks of feedback is to eliminate the dependency of solutions on initial conditions. In this case, all solutions converge to some steady-state solution that is determined only by the input of the closed-loop system. This input can be, for example, a command signal or a signal generated by a feedforward part of the controller or, as in the observer design problem, it can be the measured signal from the observed system.

In this paper, we will provide sufficient conditions for the convergence property of Lur'e-type measure differential inclusions, i.e. measure differential inclusions consisting of a linear plant and output-dependent set-valued nonlinearities in the feedback loop) and will exploit this property to tackle the tracking problem for such systems.

The outline of the paper is as follows. Section II provides a brief introduction to measure differential inclusions. Subsequently, we define the convergence property of dynamical systems in Section III. In Section IV, we present sufficient conditions for the convergence of Lur'e-type measure differential inclusions, which are exploited to provide a solution to the tracking problem in Section V. An illustrative example of a mechanical system with a unilateral constraint is discussed in detail in Section VI. Finally, Section VII presents concluding remarks. 


\section{NOTATION AND DEFINITIONS}

\section{Definition 1 (Maximal Monotone Set-valued Function)}

A set-valued function $\mathcal{F}(\boldsymbol{x}): \mathbb{R}^{n} \rightarrow \mathbb{R}^{n}$ is called monotone if its graph is monotone in the sense that for all $(\boldsymbol{x}, \boldsymbol{y}) \in$ $\operatorname{Graph}(\mathcal{F})$ and for all $\left(\boldsymbol{x}^{*}, \boldsymbol{y}^{*}\right) \in \operatorname{Graph}(\mathcal{F})$ it holds that $\left(\boldsymbol{y}-\boldsymbol{y}^{*}\right)^{T}\left(\boldsymbol{x}-\boldsymbol{x}^{*}\right) \geq 0$. In addition, if $\left(\boldsymbol{y}-\boldsymbol{y}^{*}\right)^{T}\left(\boldsymbol{x}-\boldsymbol{x}^{*}\right) \geq$ $\alpha\left\|\boldsymbol{x}-\boldsymbol{x}^{*}\right\|^{2}$ for some $\alpha>0$, then the set-valued map is strictly monotone. A monotone set-valued function $\mathcal{F}(\boldsymbol{x})$ is called maximal monotone if there exists no other monotone set-valued function whose graph strictly contains the graph of $\mathcal{F}$. If $\mathcal{F}$ is strictly monotone and maximal, then it is called strictly maximal monotone.

\section{Definition 2}

The system $\dot{\boldsymbol{x}}=\boldsymbol{A} \boldsymbol{x}+\boldsymbol{B} \boldsymbol{u}, \boldsymbol{y}=\boldsymbol{C} \boldsymbol{x}$ or the triple $(\boldsymbol{A}, \boldsymbol{B}, \boldsymbol{C})$ is said to be strictly passive if there exist an $\varepsilon>0$ and a matrix $\boldsymbol{P}=\boldsymbol{P}^{T}>0$ such that

$$
\boldsymbol{A}^{T} \boldsymbol{P}+\boldsymbol{P} \boldsymbol{A} \leq-\varepsilon \boldsymbol{I}, \boldsymbol{B}^{T} \boldsymbol{P}=\boldsymbol{C} .
$$

\section{Measure Differential InClusions}

In this section, we introduce the measure differential inclusion

$$
\mathrm{d} \boldsymbol{x} \in \mathrm{d} \boldsymbol{\Gamma}(t, \boldsymbol{x}(t))
$$

as has been proposed by Moreau [16]. The concept of differential inclusions has been extended to measure differential inclusions in order to allow for discontinuities in $\boldsymbol{x}(t)$, see e.g. [1]-[3]. With the differential inclusion $\dot{\boldsymbol{x}}(t) \in$ $\mathcal{F}(t, \boldsymbol{x}(t))$, in which $\mathcal{F}(t, \boldsymbol{x}(t))$ is a set-valued mapping, we are able to describe a non-smooth absolutely continuous time-evolution $\boldsymbol{x}(t)$. The solution $\boldsymbol{x}(t): \mathcal{I} \rightarrow \mathbb{R}^{n}$ fulfills the differential inclusion almost everywhere, because $\dot{\boldsymbol{x}}(t)$ does not exist on a Lebesgue negligible set $\mathcal{D} \subset \mathcal{I}$ of time-instances $t_{i} \in \mathcal{D}$ related to non-smooth state evolution. Instead of using the density $\dot{\boldsymbol{x}}(t)$, we can also write the differential inclusion using the differential measure:

$$
\mathrm{d} \boldsymbol{x} \in \mathcal{F}(t, \boldsymbol{x}(t)) \mathrm{d} t,
$$

which yields a measure differential inclusion (with $\mathrm{d} t$ the Lebesgue measure). The solution $\boldsymbol{x}(t)$ fulfills the measure differential inclusion (3) for all $t \in I$ because of the underlying integration process being associated with measures. Moreover, writing the dynamics in terms of a measure differential inclusion allows us to study a larger class of functions $\boldsymbol{x}(t)$, as we can let $\mathrm{d} \boldsymbol{x}$ contain parts other than the Lebesgue integrable part. In order to describe a timeevolution of bounded variation which is discontinuous at isolated time-instances, we let the differential measure $\mathrm{d} \boldsymbol{x}$ also have an atomic part:

$$
\mathrm{d} \boldsymbol{x}=\dot{\boldsymbol{x}}(t) \mathrm{d} t+\left(\boldsymbol{x}^{+}(t)-\boldsymbol{x}^{-}(t)\right) \mathrm{d} \eta,
$$

where $\mathrm{d} \eta$ is the atomic differential measure, being the sum of Dirac point measures, as defined in [7], [8], and $\boldsymbol{x}^{+}(t)=$ $\lim _{\tau \downarrow 0} \boldsymbol{x}(t+\tau), \boldsymbol{x}^{-}(t)=\lim _{\tau \uparrow 0} \boldsymbol{x}(t+\tau)$. Therefore, we extend the measure differential inclusion (3) with an atomic part as well: $\mathrm{d} \boldsymbol{x} \in \mathcal{F}(t, \boldsymbol{x}(t)) \mathrm{d} t+\mathcal{G}\left(t, \boldsymbol{x}^{-}(t), \boldsymbol{x}^{+}(t)\right) \mathrm{d} \eta$.
Here, $\mathcal{G}\left(t, \boldsymbol{x}^{-}(t), \boldsymbol{x}^{+}(t)\right)$ is a set-valued mapping, which in general depends on $t, \boldsymbol{x}^{-}(t)$ and $\boldsymbol{x}^{+}(t)$. More conveniently, and with some abuse of notation, we write the measure differential inclusion as in (2), where $\mathrm{d} \boldsymbol{\Gamma}(t, \boldsymbol{x}(t))$ is a setvalued measure function defined as

$$
\mathrm{d} \boldsymbol{\Gamma}(t, \boldsymbol{x}(t))=\mathcal{F}(t, \boldsymbol{x}(t)) \mathrm{d} t+\mathcal{G}\left(t, \boldsymbol{x}^{-}(t), \boldsymbol{x}^{+}(t)\right) \mathrm{d} \eta .
$$

The measure differential inclusion (2) has to be understood in the sense of integration and its solution $\boldsymbol{x}(t)$ is a function of locally bounded variation which fulfills $\boldsymbol{x}^{+}(t)=\boldsymbol{x}^{-}\left(t_{0}\right)+\int_{I} \boldsymbol{f}(t, \boldsymbol{x}) \mathrm{d} t+\boldsymbol{g}\left(t, \boldsymbol{x}^{-}, \boldsymbol{x}^{+}\right) \mathrm{d} \eta$, for every compact interval $I=\left[t_{0}, t\right]$, where the functions $\boldsymbol{f}(t, \boldsymbol{x})$ and $\boldsymbol{g}\left(t, \boldsymbol{x}^{-}, \boldsymbol{x}^{+}\right)$have to obey $\boldsymbol{f}(t, \boldsymbol{x}) \in \mathcal{F}(t, \boldsymbol{x})$, $\boldsymbol{g}\left(t, \boldsymbol{x}^{-}, \boldsymbol{x}^{+}\right) \in \mathcal{G}\left(t, \boldsymbol{x}^{-}(t), \boldsymbol{x}^{+}(t)\right)$. Note that for functions of locally bounded variation, the limits defining $x^{+}$and $\boldsymbol{x}^{-}$exist. Substitution of (4) in the measure differential inclusion (2), (5) gives $\dot{\boldsymbol{x}}(t) \mathrm{d} t+\left(\boldsymbol{x}^{+}(t)-\boldsymbol{x}^{-}(t)\right) \mathrm{d} \eta \in$ $\mathcal{F}(t, \boldsymbol{x}(t)) \mathrm{d} t+\mathcal{G}\left(t, \boldsymbol{x}^{-}(t), \boldsymbol{x}^{+}(t)\right) \mathrm{d} \eta$, which we can separate in the Lebesgue integrable part $\dot{\boldsymbol{x}}(t) \mathrm{d} t \in \mathcal{F}(t, \boldsymbol{x}(t)) \mathrm{d} t$, and atomic part $\left(\boldsymbol{x}^{+}(t)-\boldsymbol{x}^{-}(t)\right) \mathrm{d} \eta \in \mathcal{G}\left(t, \boldsymbol{x}^{-}(t), \boldsymbol{x}^{+}(t)\right) \mathrm{d} \eta$ from which we can retrieve $\dot{\boldsymbol{x}}(t) \in \mathcal{F}(t, \boldsymbol{x}(t))$ and the jump condition $\boldsymbol{x}^{+}(t)-\boldsymbol{x}^{-}(t) \in \mathcal{G}\left(t, \boldsymbol{x}^{-}(t), \boldsymbol{x}^{+}(t)\right)$. The latter formulation hints towards the relation with hybrid systems (or hybrid inclusions) as e.g. in [6]. We note that here the above jump condition may generally be implicit in the sense that the map $\mathcal{G}\left(t, \boldsymbol{x}^{-}(t), \boldsymbol{x}^{+}(t)\right)$ actually depends not only on $t, \boldsymbol{x}^{-}(t)$ but also on $\boldsymbol{x}^{+}(t)$, which is typically the case when e.g. modelling mechanical systems with unilateral constraints. In mechanical system with e.g. inelastic impacts the map $\mathcal{G}$ may only depend on $t$ and $\boldsymbol{x}^{+}(t)$.

It should be noted that the state $\boldsymbol{x}$ of (2) may be confined to a so-called admissible set, which we denote by $\mathcal{X}$. Here, we will assume that the measure differential inclusions under study exhibit the consistency property.

\section{Definition 3 ( [8])}

The measure differential inclusion (2) is consistent if for any initial condition taken in its admissible set, i.e. $\boldsymbol{x}_{0}=\boldsymbol{x}\left(t_{0}\right)$ is such that $\boldsymbol{x}_{0} \in \mathcal{X}$, there exists a solution in forward time that resides in the admissible domain, i.e. $\boldsymbol{x}(t) \in \mathcal{X}$ for almost all $t \geq t_{0}$.

\section{Assumption 1}

The measure differential inclusion (2) is consistent.

\section{Convergent Systems}

In this section, we briefly discuss the definition of convergence. Herein, the Lyapunov stability of solutions of (2) plays a central role. For the definition of stability of timevarying solutions we refer to [14], [17], or to [8] for the specific case of measure differential inclusions. The definitions of convergence properties presented here extend the definition given in [13].

We consider systems of the form

$$
\mathrm{d} \boldsymbol{x} \in \mathcal{F}(\boldsymbol{x}(t), \boldsymbol{k}(t)) \mathrm{d} t+\mathcal{G}\left(\boldsymbol{x}^{-}(t), \boldsymbol{x}^{+}(t), \boldsymbol{K}(t)\right) \mathrm{d} \eta,
$$

with state $\boldsymbol{x} \in \mathbb{R}^{n}$ and where $\boldsymbol{k}(t), \boldsymbol{K}(t) \in \mathbb{R}^{d}$ represent the non-impulsive and impulsive parts of the input, respectively. 
The function $\mathcal{G}\left(\boldsymbol{x}^{-}(t), \boldsymbol{x}^{+}(t), \boldsymbol{K}(t)\right)$ is assumed to be affine in $\boldsymbol{K}(t)$. In the following, we will consider the inputs $\boldsymbol{k}(t)$ : $\mathbb{R} \rightarrow \mathbb{R}^{d}$ to be in the class $\overline{\mathbb{P C}}_{d}$ of piecewise continuous inputs which are bounded on $\mathbb{R}$. Moreover, we will assume that $\boldsymbol{K}(t): \mathbb{R} \rightarrow \mathbb{R}^{d}$ is zero almost everywhere (which means that the impulsive inputs are separated in time) and bounded on $\mathbb{R}$; this class of functions will be denoted by $\boldsymbol{K}(t) \in \mathbb{M}_{d}$.

Let us formally define the property of convergence.

\section{Definition 4}

System (6) is said to be

- exponentially convergent if, for every input $k \in \overline{\mathbb{P C}}_{d}$, $\boldsymbol{K} \in \mathbb{M}_{d}$, there exists a solution $\overline{\boldsymbol{x}}_{k}(t)$ satisfying the following conditions:

(i) $\overline{\boldsymbol{x}}_{k}(t)$ is defined for almost all $t \in \mathbb{R}$

(ii) $\overline{\boldsymbol{x}}_{k}(t)$ is bounded for all $t \in \mathbb{R}$ for which it is defined,

(iii) $\overline{\boldsymbol{x}}_{k}(t)$ is globally exponentially stable.

The solution $\overline{\boldsymbol{x}}_{k}(t)$ is called a steady-state solution (where the subscript emphasizes the fact that the steady-state solution depends on the input, characterised by $\boldsymbol{k}(t)$ and $\boldsymbol{K}(t)$ ). As follows from the definition of convergence, any solution of a convergent system "forgets" its initial condition and converges to some steady-state solution. In general, the steady-state solution $\overline{\boldsymbol{x}}_{k}(t)$ may be non-unique. But for any two steady-state solutions $\overline{\boldsymbol{x}}_{k, 1}(t)$ and $\overline{\boldsymbol{x}}_{k, 2}(t)$ it holds that $\left\|\overline{\boldsymbol{x}}_{k, 1}(t)-\overline{\boldsymbol{x}}_{k, 2}(t)\right\| \rightarrow 0$ as $t \rightarrow+\infty$. At the same time, for exponentially convergent systems the steady-state solution is unique, as formulated below.

\section{Property 1 ( [14])}

If system (6) is exponentially convergent, then, for any input $\boldsymbol{k} \in \overline{\mathbb{P C}}_{d}, \boldsymbol{K} \in \mathbb{M}_{d}$, the steady-state solution $\overline{\boldsymbol{x}}_{k}(t)$ is the only solution defined and bounded for all $t \in \mathbb{R}$.

\section{Convergence Properties of LuR'e-Type MEASURE DIFFERENTIAL INCLUSIONS}

In this section we study the convergence properties for perturbed Lur'e-type measure differential inclusions of the following form:

$$
\begin{aligned}
\mathrm{d} \boldsymbol{x} & =\boldsymbol{A} \boldsymbol{x} \mathrm{d} t+\boldsymbol{B} \mathrm{d} \boldsymbol{w}(t)+\boldsymbol{D} \mathrm{d} \boldsymbol{s}, \\
\boldsymbol{y} & =\boldsymbol{C} \boldsymbol{x}, \quad-\mathrm{d} \boldsymbol{s} \in \mathcal{H}(\boldsymbol{y}) \mathrm{d} t+\mathcal{H}\left(\boldsymbol{y}^{+}\right) \mathrm{d} \eta,
\end{aligned}
$$

with $\boldsymbol{A} \in \mathbb{R}^{n \times n}, \boldsymbol{B} \in \mathbb{R}^{n \times d}, \boldsymbol{C} \in \mathbb{R}^{m \times n}, \boldsymbol{D} \in \mathbb{R}^{n \times m}$ and $\boldsymbol{x} \in \mathbb{R}^{n}$ is the system state. Moroever, $\mathrm{d} \boldsymbol{s}=\boldsymbol{\lambda} \mathrm{d} t+\boldsymbol{\Lambda} \mathrm{d} \eta$ and $\mathcal{H}(\boldsymbol{y}) \mathrm{d} t+\mathcal{H}\left(\boldsymbol{y}^{+}\right) \mathrm{d} \eta$ is the differential measure of the nonlinearity in the feedback loop that is characterised by the set-valued maximal monotone mapping $\mathcal{H}(\boldsymbol{y})$ with $\mathbf{0} \in \mathcal{H}(\mathbf{0})$. These properties of $\mathcal{H}(\boldsymbol{y})$ imply that $\boldsymbol{y}^{\mathrm{T}} \boldsymbol{h} \geq \mathbf{0}$ for all $\boldsymbol{h} \in \mathcal{H}(\boldsymbol{y})$ and $\boldsymbol{y} \in\left\{\boldsymbol{y} \in \mathbb{R}^{m} \mid \boldsymbol{y}=\boldsymbol{C} \boldsymbol{x} \wedge \boldsymbol{x} \in \mathcal{X}\right\}$, i.e. the action of $\mathcal{H}$ is passive. Furthermore, the inclusion in (7) indicates that $\boldsymbol{\lambda} \in-\boldsymbol{H}(\boldsymbol{y})$ and $\boldsymbol{\Lambda} \in-\boldsymbol{H}\left(\boldsymbol{y}^{+}\right)$. Finally, the differential measure of the time-dependent perturbation is decomposed as $\mathrm{d} \boldsymbol{w}(t)=\boldsymbol{k}(t) \mathrm{d} t+\boldsymbol{K}(t) \mathrm{d} \eta$, where $\boldsymbol{k}(t) \in \overline{\mathbb{P C}}_{d}, \boldsymbol{K}(t) \in \mathbb{M}_{d}$ are functions that represent the non-impulsive and impulsive parts of the perturbation, respectively.

In the following theorem, we state conditions under which system (7) is exponentially convergent. Later, we will exploit this property to solve the tracking problem. However, since the convergence property has been shown to be beneficial in a wider context, for example in the scope of output regulation, observer design and performance analysis for nonlinear systems, we state this result separately here.

\section{Theorem 1}

Consider a measure differential inclusion of the form (7), which satisfies Assumption 1, with $\mathcal{H}(\boldsymbol{y})$ a (set-valued) maximal monotone mapping with $\mathbf{0} \in \mathcal{H}(\mathbf{0})$. If the following conditions are satisfied:

1) the triple $(\boldsymbol{A}, \boldsymbol{D}, \boldsymbol{C})$ is strictly passive. In other words, there exists a positive definite matrix $\boldsymbol{P}=\boldsymbol{P}^{\mathrm{T}}>0$ and $\alpha>0$ for which the following conditions are satisfied:

$$
\boldsymbol{A}^{\mathrm{T}} \boldsymbol{P}+\boldsymbol{P} \boldsymbol{A} \leq-2 \alpha \boldsymbol{P}, \boldsymbol{D}^{\mathrm{T}} \boldsymbol{P}=\boldsymbol{C}
$$

2) there exists a $\beta \in \mathbb{R}$ such that $\left(\boldsymbol{x}^{+}\right)^{\mathrm{T}} \boldsymbol{P} \boldsymbol{B} \boldsymbol{K}(t) \leq \beta$ for all $\boldsymbol{x} \in \mathcal{X}$ and $\boldsymbol{P}$ satisfying (8); i.e. the energy input of the impulsive inputs is bounded from above,

3) the time instances $t_{i}$ for which the input is impulsive, i.e. for which $\boldsymbol{K}(t)$ is non-zero, are separated by the dwell-time $\tau \leq t_{i+1}-t_{i}$, with

$$
\begin{aligned}
& \tau=\frac{\delta}{2(\delta-1) \alpha} \ln \left(1+\frac{2 \beta}{\delta^{2} \gamma^{2}}\right), \\
& \gamma:=\sup _{t \in \mathbb{R}, \boldsymbol{\lambda}(\mathbf{0}) \in-\mathcal{H}(\mathbf{0})}\left\{\frac{\|\boldsymbol{B} \boldsymbol{k}(t)+\boldsymbol{D} \boldsymbol{\lambda}(\mathbf{0})\|_{P}}{\alpha}\right\},
\end{aligned}
$$

for some $\delta>1$.

then the system (7), with inputs $\boldsymbol{k}(t)$ and $\boldsymbol{K}(t)$, is exponentially convergent.

Proof: The proof is given in the appendix.

\section{Tracking Control Problem}

In this section we study the tracking control problem for Lur'e-type measure differential inclusions of the following form:

$$
\begin{aligned}
\mathrm{d} \boldsymbol{x} & =\boldsymbol{A}_{o l} \boldsymbol{x} \mathrm{d} t+\boldsymbol{B} \mathrm{d} \boldsymbol{u}+\boldsymbol{D} \mathrm{d} s, \\
\boldsymbol{y} & =\boldsymbol{C} \boldsymbol{x}, \quad-\mathrm{d} \boldsymbol{s} \in \mathcal{H}(\boldsymbol{y}) \mathrm{d} t+\boldsymbol{H}\left(\boldsymbol{y}^{+}\right) \mathrm{d} \eta,
\end{aligned}
$$

with $\boldsymbol{A}_{o l} \in \mathbb{R}^{n \times n}$ and $\mathrm{d} \boldsymbol{u}=\boldsymbol{p} \mathrm{d} t+\boldsymbol{P} \mathrm{d} \eta$ is the differential measure of the control action.

The tracking problem considered in this work is formalised as follows:

\section{Tracking problem:}

Design a control law for $\mathrm{d} \boldsymbol{u}$ that, based on information on the desired state trajectory $\boldsymbol{x}_{d}(t)$ and the measured state $\boldsymbol{x}$, renders $\boldsymbol{x}(t) \rightarrow \boldsymbol{x}_{d}(t)$ as $t \rightarrow \infty$ and the states of the closed-loop system are bounded.

To solve this problem, we adopt the following assumption: 


\section{Assumption 2}

The desired trajectory $\boldsymbol{x}_{d}(t)$ is a function of locally bounded variation and there exists $\mathrm{d} \boldsymbol{u}_{f f}(t)=\boldsymbol{p}_{f f}(t) \mathrm{d} t+\boldsymbol{P}_{f f}(t) \mathrm{d} \eta$, with both $\boldsymbol{p}_{f f}(t) \in \overline{\mathbb{P C}}_{d}$ and $\boldsymbol{P}_{f f}(t) \in \mathbb{M}_{d}$, such that $\boldsymbol{x}_{d}(t)$ satisfies

$$
\begin{aligned}
\mathrm{d} \boldsymbol{x}_{d}(t) & =\boldsymbol{A}_{o l} \boldsymbol{x}_{d}(t) \mathrm{d} t+\boldsymbol{B} \mathrm{d} \boldsymbol{u}_{f f}(t)+\boldsymbol{D} \mathrm{d} \boldsymbol{s}, \\
-\mathrm{d} \boldsymbol{s} & \in \mathrm{d} \boldsymbol{H}\left(\boldsymbol{C} \boldsymbol{x}_{d}(t)\right) \mathrm{d} t+\boldsymbol{H}\left(\boldsymbol{C} \boldsymbol{x}_{d}^{+}(t)\right) \mathrm{d} \eta,
\end{aligned}
$$

i.e. $\mathrm{d} \boldsymbol{u}_{f f}(t)$ can be considered to be a reference control (feedforward) generating $\boldsymbol{x}_{d}(t)$.

When addressing the tracking problem, it is commonly split in two parts: firstly, finding the appropriate feedforward and, secondly, stabilising the desired solution. In the current paper, we primarily focus on the second problem. Note that also for smooth systems the existence of the feedforward is a natural assumption (think of the solvability of the regulator equations as a natural assumption in the scope of output regulation [14], [18]).

We propose to tackle the tracking problem by means of a combination of Lebesgue measurable linear error-feedback and a possibly impulsive feedforward control:

$$
\mathrm{d} \boldsymbol{u}=\boldsymbol{u}_{f b}\left(\boldsymbol{x}, \boldsymbol{x}_{d}(t)\right) \mathrm{d} t+\mathrm{d} \boldsymbol{u}_{f f}(t),
$$

with $\boldsymbol{u}_{f b}\left(\boldsymbol{x}, \boldsymbol{x}_{d}(t)\right)=\boldsymbol{N}\left(\boldsymbol{x}-\boldsymbol{x}_{d}(t)\right), \quad \mathrm{d} \boldsymbol{u}_{f f}(t)=$ $\boldsymbol{p}_{f f}(t) \mathrm{d} t+\boldsymbol{P}_{f f}(t) \mathrm{d} \eta$, where $\boldsymbol{N} \in \mathbb{R}^{d \times n}$ is the feedback gain matrix. We restrict the energy input of the impulsive control action $\boldsymbol{P}_{f f}(t)$ to be bounded from above: $\left(\boldsymbol{x}^{+}\right)^{\mathrm{T}} \boldsymbol{B} \boldsymbol{P}_{f f} \leq \beta$. Note that this condition puts a bound on the jumps in the desired trajectory $\boldsymbol{x}_{d}(t)$ which can be realised. Combining the control law (12) with the system dynamics (10) yields the closed-loop dynamics:

$$
\begin{aligned}
\mathrm{d} \boldsymbol{x} & =\boldsymbol{A} \boldsymbol{x} \mathrm{d} t+\boldsymbol{D} \mathrm{d} s+\boldsymbol{B}\left(-\boldsymbol{N} \boldsymbol{x}_{d}(t) \mathrm{d} t+\mathrm{d} \boldsymbol{u}_{f f}(t)\right), \\
\boldsymbol{y} & =\boldsymbol{C} \boldsymbol{x}, \quad-\mathrm{d} \boldsymbol{s} \in \boldsymbol{H}(\boldsymbol{y}) \mathrm{d} t+\boldsymbol{H}\left(\boldsymbol{y}^{+}\right) \mathrm{d} \eta,
\end{aligned}
$$

with $\boldsymbol{A}=\boldsymbol{A}_{\text {ol }}+\boldsymbol{B} \boldsymbol{N}$. In the next result, the convergence property of the closed-loop system is exploited to solve the tracking problem.

\section{Theorem 2}

Consider a measure differential inclusion of the form (10), which satisfies Assumption 1, with $\mathcal{H}(\boldsymbol{y})$ a (set-valued) maximal monotone mapping with $\mathbf{0} \in \mathcal{H}(\mathbf{0})$. Suppose the desired trajectory $\boldsymbol{x}_{d}(t)$ satisfies Assumption 2 with $\mathrm{d} \boldsymbol{u}_{f f}(t)$ being the corresponding feedforward. If the conditions in Theorem 1 are satisfied, with $\boldsymbol{k}(t):=-\boldsymbol{K} \boldsymbol{x}_{d}(t)+\boldsymbol{p}_{f f}(t)$ and $\boldsymbol{K}(t):=\boldsymbol{P}_{f f}(t)$, then the desired solution $\boldsymbol{x}_{d}(t)$ is a globally exponentially stable solution of the closed-loop system (13), i.e. the tracking problem is solved.

Proof: Since the conditions of Theorem 1 are satisfied, all solutions of the closed-loop system (13) converge to each other exponentially, see (21) in the proof of Theorem 1. Since the desired solution is a solution of (13), for $\boldsymbol{x}(0)=\boldsymbol{x}_{d}(0)$, by the choice of the feedforward, see Assumption 2, the desired solution is a globally exponentially stable solution of (13).

\section{EXAMPLE OF A MECHANICAL SYSTEM WITH A UNILATERAL CONSTRAINT}

Let us consider a mechanical system consisting of two inertias, $m_{1}$ and $m_{2}$, which are coupled by a linear spring $c$ and a linear damper $b_{1}$, see Figure 1 . The inertia $m_{1}$ is attached to the earth by a linear damper $b_{2}$ and $m_{2}$ is subject to a one-way clutch. Moreover, $m_{1}$ is actuated by a (possibly impulsive) control force $\mathrm{d} u$. The open-loop dynamics is

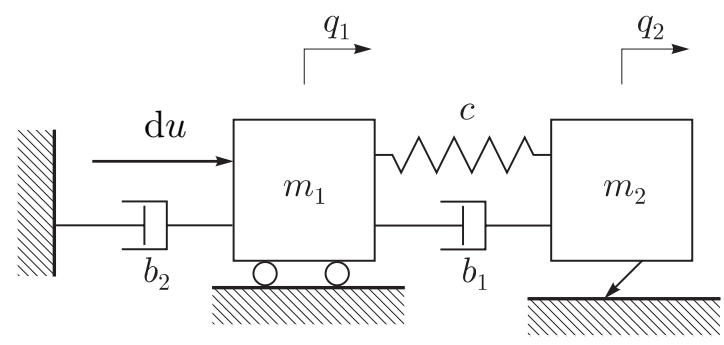

Fig. 1: Motor-load configuration with one-way clutch and impulsive actuation.

described by (10) with

$$
\boldsymbol{A}_{o l}=\left[\begin{array}{ccc}
0 & -1 & 1 \\
\frac{c}{m_{1}} & -\frac{b_{1}+b_{2}}{m_{1}} & \frac{b_{1}}{m_{1}} \\
-\frac{c}{m_{2}} & \frac{b_{1}}{m_{2}} & -\frac{b_{1}}{m_{2}}
\end{array}\right], \quad \boldsymbol{B}=\left[\begin{array}{c}
0 \\
\frac{1}{m_{1}} \\
0
\end{array}\right],
$$

$\boldsymbol{D}^{\mathrm{T}}=\left[\begin{array}{lll}0 & 0 & \frac{1}{m_{2}}\end{array}\right]$ and $\boldsymbol{C}=\left[\begin{array}{lll}0 & 0 & 1\end{array}\right]$. The state vector is given by $\boldsymbol{x}=\left[\begin{array}{lll}q_{2}-q_{1} & u_{1} & u_{2}\end{array}\right]^{\mathrm{T}}$, with $q_{1}$ and $q_{2}$ the displacements of $m_{1}$ and $m_{2}$, respectively, and $u_{1}$ and $u_{2}$ the velocities of $m_{1}$ and $m_{2}$, respectively. The differential measure $\mathrm{d} s=\mathcal{H}(\boldsymbol{y}) \mathrm{d} t+\mathcal{H}\left(\boldsymbol{y}^{+}\right) \mathrm{d} \eta$ of the force in the oneway clutch is characterised by the scalar set-valued maximal monotone mapping $\mathcal{H}(x)=\operatorname{Upr}(x)$. The set-valued function $\operatorname{Upr}(x)$ is the unilateral primitive [7]: $-y \in \operatorname{Upr}(x) \Leftrightarrow$ $0 \leq x \perp y \geq 0 \Leftrightarrow x \geq 0, y \geq 0, x y=0$, being a maximal monotone operator. We adopt the following system parameters: $m_{1}=m_{2}=1, c=10, b_{1}=1$ and $b_{2}=-1.4$.

The desired velocity of the second mass is a periodic sawtooth wave with period time $T$ :

$x_{d 3}(t)=\left\{\begin{array}{cc}\bmod (t, T) & \text { for } 0 \leq \bmod (t, T) \leq \frac{T}{4} \\ \frac{T}{2}-\bmod (t, T) & \text { for } \frac{T}{4} \leq \bmod (t, T) \leq \frac{T}{2} \\ 0 & \text { for } \frac{T}{2} \leq \bmod (t, T) \leq T\end{array}\right.$,

where these equations represent a ramp-up, ramp-down, and a deadband phase, respectively. The signal $x_{d 3}(t)$ for $T=1 \mathrm{~s}$ is shown by the dotted line in Fig. 2. The desired trajectory $x_{d 3}(t)$ is a periodic signal which is time-continuous but has three kinks in each period. Kinks in $x_{d 3}(t)$ can be achieved by applying an impulsive force on the first mass which causes an instantaneous change in the velocity $x_{2}=u_{1}$ and therefore a discontinuous force in the damper $b_{1}$. The oneway clutch on the second mass prevents negative values of $x_{d 3}$ and no impulsive force on the first mass is therefore necessary for the change from ramp-down to deadband. In a 
first step, the signals $x_{d 1}(t), x_{d 2}(t)$ and $\mathrm{d} s(t)$ are designed such that

$$
\begin{aligned}
\dot{x}_{d 1}(t)= & -x_{d 2}(t)+x_{d 3}(t) \\
\mathrm{d} x_{d 3}(t)= & \left(-\frac{c}{m_{2}} x_{d 1}(t)-\frac{b_{1}}{m_{2}}\left(-x_{d 2}(t)+x_{d 3}(t)\right)\right) \mathrm{d} t \\
& +\frac{1}{m_{2}} \mathrm{~d} s(t),
\end{aligned}
$$

with

$$
-\mathrm{d} s(t) \in \operatorname{Upr}\left(x_{d 3}(t)\right) \mathrm{d} t+\operatorname{Upr}\left(x_{d 3}^{+}(t)\right) \mathrm{d} \eta,
$$

for the given periodic trajectory $x_{d 3}(t)$. The solution of this problem is not unique as we are free to chose $\mathrm{d} s(t) \geq 0$ for $x_{d 3}(t)=0$. By fixing $d s(t)=\dot{s}_{0} \mathrm{~d} t$ to a constant value for $x_{d 3}(t)=0$ (i.e. $\dot{s}_{0}$ is a constant), we obtain the following discontinuous differential equation for $x_{d 1}(t)$ :

$\dot{x}_{d 1}= \begin{cases}\frac{m_{2}}{b_{1}}\left(-\dot{x}_{d 3}(t)-\frac{c}{m_{2}} x_{d 1}\right) & x_{d 3}(t)>0 \\ \frac{m_{2}}{b_{1}}\left(-\dot{x}_{d 3}(t)-\frac{c}{m_{2}} x_{d 1}+\frac{1}{m_{2}} \dot{s}_{0}\right) & x_{d 3}(t)=0 .\end{cases}$

The numerical solution of $x_{d 1}(t)$ gives (after a transient) a periodic signal $x_{d 1}(t)$ and $x_{d 2}(t)=-\dot{x}_{d 1}(t)+x_{d 3}(t)$ (see the dotted lines in Figs. 4 and 5 which are mostly hidden by the solid lines). We have taken $\dot{s}_{0}=1$. Subsequently, the feedforward input $\mathrm{d} u_{f f}=p_{f f} \mathrm{~d} t+P_{f f} \mathrm{~d} \eta$ is designed such that

$$
\mathrm{d} u_{f f}=m_{1} \mathrm{~d} x_{d 2}-\left(c x_{d 1}+b_{1}\left(-x_{d 2}+x_{d 3}\right)-b_{2} x_{d 2}\right) \mathrm{d} t
$$

and it therefore holds that $\boldsymbol{x}(t)=\boldsymbol{x}_{d}(t)$ for $t \geq 0$ if $\boldsymbol{x}(0)=$ $\boldsymbol{x}_{d}(0)$, where $\boldsymbol{x}(t)$ is a solution of (10), (14), with $\mathrm{d} u=$ $\mathrm{d} u_{f f}$. The feedforward input $\mathrm{d} u_{f f} / \mathrm{d} t$ is shown in Fig. 6 and is equal to $p_{f f}(t)$ almost everywhere. Two impulsive inputs $P_{f f}(t)$ per period can be seen at the time-instances for which there is a change from 'ramp-up to ramp-down' and from 'deadband to ramp-up'. Next, we implement the control law (12) on system (10) with the feedforward $\mathrm{d} u_{f f}$ as in (17). We choose $\boldsymbol{N}=\left[\begin{array}{lll}0 & -4 & 0\end{array}\right]$ which ensures that (8) is satisfied with

$$
\boldsymbol{P}=\left[\begin{array}{ccc}
34 & -10.5 & 0 \\
-10.5 & 6 & 0 \\
0 & 0 & 1
\end{array}\right], \quad \alpha=0.25
$$

Consequently, the closed-loop system (10), (14), (12), (17) is exponentially convergent. Fig. 2 shows the closed-loop dynamics for which the desired periodic solution $\boldsymbol{x}_{d}(t)$ is globally exponentially stable. Fig. 3 shows the open-loop dynamics for which there is no state-feedback. Without feedback, the desired periodic solution $\boldsymbol{x}_{d}(t)$ is not globally attractive, not even locally, and the solution from the chosen initial condition is attracted to a stable period- 2 solution. Clearly, the system without feedback is not convergent. For both cases the initial condition $\boldsymbol{x}(0)=\left[\begin{array}{lll}0.16 & 2.17 & 0\end{array}\right]^{\mathrm{T}}$ was used. Figs. 4 and 5 show the time-histories of $x_{1}(t)$ and $x_{d 1}(t)$, respectively $x_{2}(t)$ and $x_{d 2}(t)$, in solid and dotted lines. Jumps in the state $x_{2}(t)$ and desired state $x_{d 2}(t)$ can be seen on time-instances for which the feedforward input is impulsive.

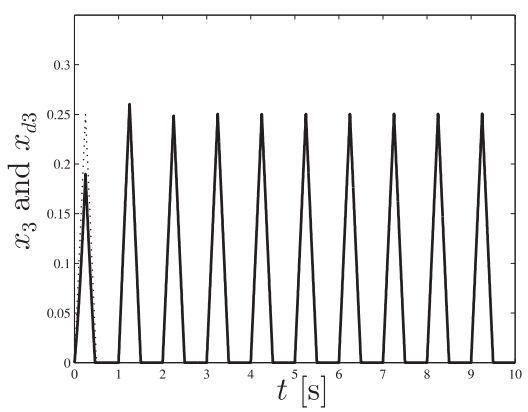

Fig. 2: $x_{3}(t)$ (solid) and $x_{d 3}(t)$ (dotted) for the case of feedback and feedforward control.

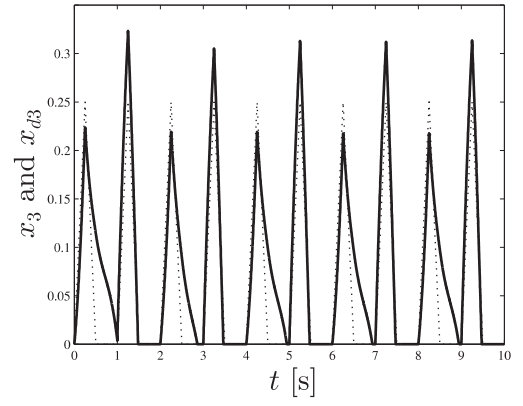

Fig. 3: $x_{3}(t)$ (solid) and $x_{d 3}(t)$ (dotted) for the case of only feedforward control.

\section{CONCLUSIONS}

In this paper, the tracking problem for Lur'e-type measure differential inclusions is studied. The framework of measure differential inclusions allows us to describe systems with discontinuities in the state evolution, such as mechanical systems with unilateral constraints. In the scope of the tracking problem, the stability properties of time-varying solutions play a central role. Therefore, we have presented results on the stability of time-varying solutions of such systems in the terms of the convergence property. Next, this property is exploited to provide a solution to the tracking problem, where the desired solution may exhibit state jumps. The results are illustrated by application to a mechanical motion system with a unilateral velocity constraint.

\section{REFERENCES}

[1] M. Monteiro Marques, Differential Inclusions in Nonsmooth Mechanical Systems. Basel: Birkhaüser, 1993.

[2] J. J. Moreau, "Unilateral contact and dry friction in finite freedom dynamics," in Non-Smooth Mechanics and Applications, ser. CISM Courses and Lectures, J. J. Moreau and P. D. Panagiotopoulos, Eds. Wien: Springer, 1988, vol. 302, pp. 1-82.

[3] B. Brogliato, Nonsmooth Mechanics, 2nd ed. London: Springer, 1999.

[4] V. Acary, B. Brogliato, and D. Goeleven, "Higher order Moreaus sweeping process: mathematical formulation and numerical simulation," Mathematical Programming, Series A, vol. 113, pp. 133-217, 2008.

[5] A. J. van der Schaft and J. M. Schumacher, An Introduction to Hybrid Dynamical Systems, ser. Lecture Notes in Control and Information Sciences. London: Springer, 2000, vol. 251.

[6] R. Goebel and A. Teel, "Solutions to hybrid inclusions via set and graphical convergence with stability theory applications," Automatica, vol. 42, no. 4, pp. 573-587, 2006.

[7] Ch. Glocker, Set-Valued Force Laws, Dynamics of Non-Smooth Systems, ser. Lecture Notes in Applied Mechanics. Berlin: SpringerVerlag, 2001, vol. 1. 


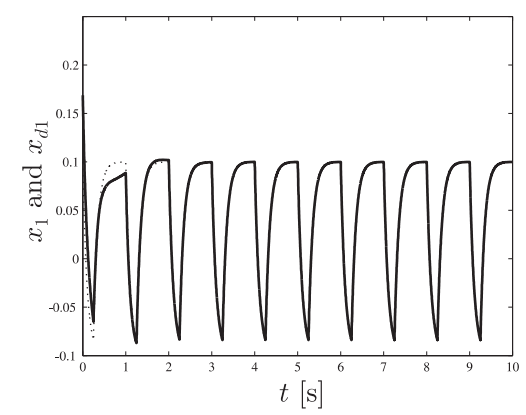

Fig. 4: $x_{1}(t)$ (solid) and $x_{d 1}(t)$ (dotted) for the case of feedback and feedforward control.

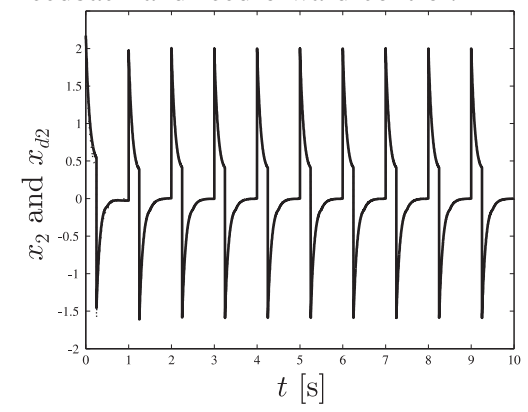

Fig. 5: $x_{2}(t)$ (solid) and $x_{d 2}(t)$ (dotted) for the case of feedback and feedforward control.

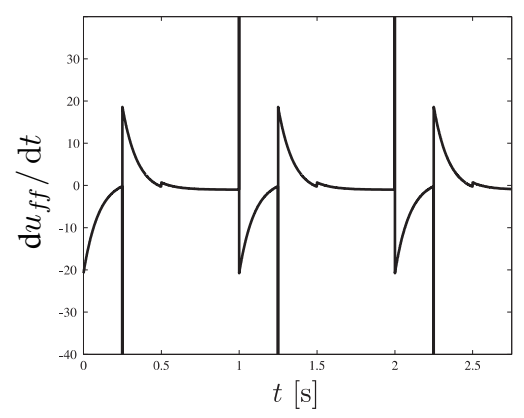

Fig. 6: Feedforward $\mathrm{d} u_{f f} / \mathrm{d} t$.

[8] R. I. Leine and N. van de Wouw, Stability and Convergence of Mechanical Systems with Unilateral Constraints, ser. Lecture Notes in Applied and Computational Mechanics. Berlin: Springer Verlag, 2008, vol. 36.

[9] B. Brogliato, "Absolute stability and the Lagrange-Dirichlet theorem with monotone multivalued mappings," Systems \& Control Letters, vol. 51, pp. 343-353, 2004.

[10] J. M. Bourgeot and B. Brogliato, "Tracking control of complementarity Lagrangian systems," Int. J. Bifurcation and Chaos, vol. 15, no. 6, pp. 1839-1866, 2005.

[11] B. Brogliato, S.-I. Niculescu, and P. Orhant, "On the control of finitedimensional mechanical systems with unilateral constraints," IEEE Trans. on Automatic Control, vol. 42, no. 2, pp. 200-215, 1997.

[12] L. Menini and A. Tornambè, "Asymptotic tracking of periodic trajectories for a simple mechanical system subject to nonsmooth impacts," IEEE Trans. on Automatic Control, vol. 46, no. 7, pp. 1122-1126, 2001.

[13] B. P. Demidovich, Lectures on Stability Theory (in Russian). Moscow: Nauka, 1967.

[14] A. Pavlov, N. van de Wouw, and H. Nijmeijer, Uniform Output Regulation of Nonlinear Systems: A Convergent Dynamics Approach. Boston: Birkhäuser, 2005, in Systems \& Control: Foundations and Applications (SC) Series.

[15] - "Convergent dynamics, a tribute to B.P. Demidovich," Systems and Control Letters, vol. 52, no. 3-4, pp. 257-261, 2004.
[16] J. J. Moreau, "Bounded variation in time," in Topics in Nonsmooth Mechanics, J. J. Moreau, P. D. Panagiotopoulos, and G. Strang, Eds. Basel, Boston, Berlin: Birkhäuser Verlag, 1988, pp. 1-74.

[17] J. L. Willems, Stability theory of Dynamical Systems. London: Thomas Nelson and Sons Ltd., 1970.

[18] A. Isidori and C. I. Byrnes, "Output regulation of nonlinear systems," IEEE Trans. on Automatic Control, vol. 35, pp. 131-140, 1990.

[19] V. Yakubovich, "Matrix inequalities method in stability theory for nonlinear control systems: I. absolute stability of forced vibrations," Automation and Remote Control, vol. 7, pp. 905-917, 1964.

\section{APPENDIX}

In this proof, we will show that system (7) is exponentially convergent. Hereto, we first show that the all solutions of the system converge to each other exponentially. The next step in the proof of exponential convergence is to show that there exists a unique (steady-state) solution that is bounded on $t \in \mathbb{R}$.

Consider two solutions $\boldsymbol{x}_{1}(t)$ and $\boldsymbol{x}_{2}(t)$ of the closedloop system (7) and a Lyapunov candidate function $V=$ $\frac{1}{2}\left\|\boldsymbol{x}_{2}-\boldsymbol{x}_{1}\right\|_{P}^{2}$, where we adopt the notation $\|\boldsymbol{\xi}\|_{P}^{2}=\boldsymbol{\xi}^{\mathrm{T}} \boldsymbol{P} \boldsymbol{\xi}$. Consequently, the differential measure of $V$ satisfies: $\mathrm{d} V=$ $\frac{1}{2}\left(\boldsymbol{x}_{2}^{+}+\boldsymbol{x}_{2}^{-}-\boldsymbol{x}_{1}^{+}-\boldsymbol{x}_{1}^{-}\right)^{\mathrm{T}} \boldsymbol{P}\left(\mathrm{d} \boldsymbol{x}_{2}-\mathrm{d} \boldsymbol{x}_{1}\right)$, with $\mathrm{d} \boldsymbol{x}_{i}=$ $\boldsymbol{A} \boldsymbol{x}_{i} \mathrm{~d} t+\boldsymbol{D} \mathrm{d} s_{i}+\boldsymbol{B} \mathrm{d} \boldsymbol{w}(t), \quad i=1,2$, where $\mathrm{d} \boldsymbol{s}_{i}=$ $\boldsymbol{\lambda}_{i} \mathrm{~d} t+\boldsymbol{\Lambda}_{i} \mathrm{~d} \eta$, with $\boldsymbol{\lambda}_{i} \in-\boldsymbol{H}\left(\boldsymbol{C} \boldsymbol{x}_{i}\right), \boldsymbol{\Lambda}_{i} \in-\boldsymbol{H}\left(\boldsymbol{C} \boldsymbol{x}_{i}^{+}\right)$, $i=1,2$. The differential measure of $V$ has a density $\dot{V}$ with respect to the Lebesgue measure $\mathrm{d} t$ and a density $V^{+}-V^{-}$with respect to the atomic differential measure $\mathrm{d} \eta$, i.e. $\mathrm{d} V=\dot{V} \mathrm{~d} t+\left(V^{+}-V^{-}\right) \mathrm{d} \eta$. We first evaluate the density $\dot{V}$ :

$$
\begin{aligned}
\dot{V}= & \left(\boldsymbol{x}_{2}-\boldsymbol{x}_{1}\right)^{\mathrm{T}} \boldsymbol{P}\left(\boldsymbol{D} \boldsymbol{\lambda}_{2}+\boldsymbol{A} \boldsymbol{x}_{2}-\left(\boldsymbol{D} \boldsymbol{\lambda}_{1}+\boldsymbol{A} \boldsymbol{x}_{1}\right)\right) \\
= & \frac{1}{2}\left(\boldsymbol{x}_{2}-\boldsymbol{x}_{1}\right)^{\mathrm{T}} \\
& \quad\left(\left(\boldsymbol{P} \boldsymbol{A}+\boldsymbol{A}^{\mathrm{T}} \boldsymbol{P}\right)\left(\boldsymbol{x}_{2}-\boldsymbol{x}_{1}\right)+2 \boldsymbol{C}^{\mathrm{T}}\left(\boldsymbol{\lambda}_{2}-\boldsymbol{\lambda}_{1}\right)\right), \\
\leq & -\alpha\left\|\boldsymbol{x}_{2}-\boldsymbol{x}_{1}\right\|_{P}^{2},
\end{aligned}
$$

where we used that both solutions $\boldsymbol{x}_{1}$ and $\boldsymbol{x}_{2}$ correspond to the same perturbation $\mathrm{d} \boldsymbol{w}(t)$, the fact that (8) is satisfied and the fact that the mapping $\mathcal{H}(\boldsymbol{y})$ is monotone. Subsequently, we consider the jump $V^{+}-V^{-}$of $V: V^{+}-V^{-}=\frac{1}{2}\left(\boldsymbol{x}_{2}^{+}+\right.$ $\left.\boldsymbol{x}_{2}^{-}-\boldsymbol{x}_{1}^{+}-\boldsymbol{x}_{1}^{-}\right)^{\mathrm{T}} \boldsymbol{P}\left(\boldsymbol{x}_{2}^{+}-\boldsymbol{x}_{2}^{-}-\boldsymbol{x}_{1}^{+}+\boldsymbol{x}_{1}^{-}\right)$, with $\boldsymbol{x}_{i}^{+}-\boldsymbol{x}_{i}^{-}=$ $\boldsymbol{D} \boldsymbol{\Lambda}_{i}+\boldsymbol{B} \boldsymbol{K}(t), \quad \boldsymbol{\Lambda}_{i} \in-\mathcal{H}\left(\boldsymbol{C} \boldsymbol{x}_{i}^{+}\right), i=1,2$. Elimination of $\boldsymbol{x}_{1}^{-}$and $\boldsymbol{x}_{2}^{-}$and exploiting the monotonicity of $\mathcal{H}(\boldsymbol{y})$ gives

$$
\begin{aligned}
& V^{+}-V^{-}=\left(\boldsymbol{x}_{2}^{+}-\boldsymbol{x}_{1}^{+}-\frac{\boldsymbol{D}}{2}\left(\boldsymbol{\Lambda}_{2}-\boldsymbol{\Lambda}_{1}\right)\right)^{\mathrm{T}} \boldsymbol{P} \boldsymbol{D}\left(\boldsymbol{\Lambda}_{2}-\boldsymbol{\Lambda}_{1}\right) \\
& =\left(\boldsymbol{y}_{2}^{+}-\boldsymbol{y}_{1}^{+}\right)^{\mathrm{T}}\left(\boldsymbol{\Lambda}_{2}-\boldsymbol{\Lambda}_{1}\right)-\frac{1}{2}\left\|\left(\boldsymbol{D} \boldsymbol{\Lambda}_{2}-\boldsymbol{D} \boldsymbol{\Lambda}_{1}\right)\right\|_{P}^{2} \leq 0,
\end{aligned}
$$

where we used the matrix equality in (8). Using (19) and (20), the differential measure of $V$ satisfies $\mathrm{d} V \leq$ $-2 \alpha V \mathrm{~d} t$, along solutions of (7). It therefore holds that $V$ strictly decreases (exponentially) over every Lebesgue non-negligible time-interval as long as $\boldsymbol{x}_{2} \neq \boldsymbol{x}_{1}$. In turn, this implies that all solutions of (7) converge to each other 
exponentially (i.e. the system is exponentially incrementally stable):

$$
\begin{aligned}
& \left\|\boldsymbol{x}_{2}^{+}(t)-\boldsymbol{x}_{1}^{+}(t)\right\| \\
& \quad \leq \sqrt{\frac{\lambda_{\max }(\boldsymbol{P})}{\lambda_{\min }(\boldsymbol{P})}} e^{-\alpha\left(t-t_{0}\right)}\left\|\boldsymbol{x}_{2}^{-}\left(t_{0}\right)-\boldsymbol{x}_{1}^{-}\left(t_{0}\right)\right\|,
\end{aligned}
$$

for $t \geq t_{0}$ and where $\lambda_{\max }(\boldsymbol{P})$ and $\lambda_{\min }(\boldsymbol{P})$ represent the maximum and minimum eigenvalue of $\boldsymbol{P}$, respectively.

Let us now show that there exists a unique (steady-state) solution that is bounded on $t \in \mathbb{R}$. Consider, hereto, the Lyapunov candidate function $W=\frac{1}{2} \boldsymbol{x}^{\mathrm{T}} \boldsymbol{P} \boldsymbol{x}$. The differential measure of $W$ can be decomposed as $\mathrm{d} W=\dot{W} \mathrm{~d} t+\left(W^{+}{ }_{-}\right.$ $\left.W^{-}\right) \mathrm{d} \eta$. We first evaluate the density $\dot{W}$ :

$$
\begin{aligned}
\dot{W}= & \boldsymbol{x}^{\mathrm{T}} \boldsymbol{P}(\boldsymbol{D} \boldsymbol{\lambda}+\boldsymbol{A} \boldsymbol{x}+\boldsymbol{B} \boldsymbol{k}(t)) \\
= & \boldsymbol{x}^{\mathrm{T}} \boldsymbol{P} \boldsymbol{D}(\boldsymbol{\lambda}-\boldsymbol{\lambda}(\mathbf{0}))+\frac{1}{2} \boldsymbol{x}^{\mathrm{T}}\left(\boldsymbol{P} \boldsymbol{A}+\boldsymbol{A}^{\mathrm{T}} \boldsymbol{P}\right) \boldsymbol{x} \\
& +\boldsymbol{x}^{T} \boldsymbol{P} \boldsymbol{B} \boldsymbol{k}(t)+\boldsymbol{x}^{\mathrm{T}} \boldsymbol{P} \boldsymbol{D} \boldsymbol{\lambda}(\mathbf{0}),
\end{aligned}
$$

with $\boldsymbol{\lambda} \in \mathcal{H}(\boldsymbol{y})$ and $\boldsymbol{\lambda}(\mathbf{0}) \in \mathcal{H}(\mathbf{0})$. Due to the satisfaction of (8) and the monotonicity of $\mathcal{H}(\boldsymbol{y})$, we have that $\dot{W} \leq$ $-\alpha\|\boldsymbol{x}\|_{P}^{2}+\|\boldsymbol{x}\|_{P}\|\boldsymbol{B} \boldsymbol{k}(t)+\boldsymbol{D} \boldsymbol{\lambda}(\mathbf{0})\|_{P}$. Note that $\dot{W}<0$ for $\boldsymbol{x}$ satisfying $\|\boldsymbol{x}\|_{P}>\gamma$ with $\gamma$ defined in (9). Let us use the fact that the function $-\left(1-\frac{1}{\delta}\right) \alpha\|\boldsymbol{x}\|_{P}^{2}>-\alpha\|\boldsymbol{x}\|_{P}^{2}+\gamma \alpha\|\boldsymbol{x}\|_{P}$ for $\|\boldsymbol{x}\|_{P}>\delta \gamma$, where $\delta>1$ is an arbitrary constant and $\gamma>0$. It therefore holds that

$$
\dot{W} \leq-2\left(1-\frac{1}{\delta}\right) \alpha W \quad \text { for }\|\boldsymbol{x}\|_{P} \geq \delta \gamma, \delta>1 .
$$

Subsequently, we consider the jump $W^{+}-W^{-}$of $W: W^{+}-$ $W^{-}=\frac{1}{2}\left(\boldsymbol{x}^{+}+\boldsymbol{x}^{-}\right)^{\mathrm{T}} \boldsymbol{P}\left(\boldsymbol{x}^{+}-\boldsymbol{x}^{-}\right)$, with $\boldsymbol{x}^{+}-\boldsymbol{x}^{-}=\boldsymbol{D} \boldsymbol{\Lambda}+$ $\boldsymbol{B} \boldsymbol{K}(t)$ and $\boldsymbol{\Lambda} \in-\boldsymbol{H}\left(\boldsymbol{C} \boldsymbol{x}^{+}\right)$. Elimination of $\boldsymbol{x}^{-}$, exploiting the passivity of $\mathcal{H}(\boldsymbol{y})$ and using the matrix equality in (8) gives

$$
\begin{aligned}
W^{+} & -W^{-}=\frac{1}{2}\left(2 \boldsymbol{x}^{+}-\boldsymbol{D} \boldsymbol{\Lambda}-\boldsymbol{B} \boldsymbol{K}(t)\right)^{\mathrm{T}} \boldsymbol{P}(\boldsymbol{D} \boldsymbol{\Lambda}+\boldsymbol{B} \boldsymbol{K}(t)) \\
& =\left(\boldsymbol{x}^{+}\right)^{\mathrm{T}}(\boldsymbol{P} \boldsymbol{D} \boldsymbol{\Lambda}+\boldsymbol{P} \boldsymbol{B} \boldsymbol{K}(t))-\frac{1}{2}\|\boldsymbol{D} \boldsymbol{\Lambda}+\boldsymbol{B} \boldsymbol{K}(t)\|_{P}^{2} \\
& \leq \boldsymbol{y}^{+^{\mathrm{T}}} \boldsymbol{\Lambda}+\boldsymbol{x}^{+^{\mathrm{T}}} \boldsymbol{P} \boldsymbol{B} \boldsymbol{K}(t) \leq \beta,
\end{aligned}
$$

in which we used condition 2 in the theorem. Then, due to (23), for the non-impulsive part of the motion it holds that if $\left\|\boldsymbol{x}\left(t_{0}\right)\right\|_{P} \leq \delta \gamma$ then $\|\boldsymbol{x}(t)\|_{P} \leq \delta \gamma$ for all $t \in$ $\left[t_{0}, t^{*}\right]$ (if no state resets occur in this time interval). Moreover, as far as the state resets are concerned, (24) shows that a state reset from a state $x^{-}\left(t_{i}\right) \in \mathcal{V}$ with $\mathcal{V}=$ $\left\{\boldsymbol{x} \in \mathcal{X} \mid\|\boldsymbol{x}\|_{P} \leq \delta \gamma\right\}$ can only occur to $\boldsymbol{x}^{+}\left(t_{i}\right)$ such that $W\left(\boldsymbol{x}^{+}\left(t_{i}\right)\right):=\frac{1}{2}\left\|\boldsymbol{x}^{+}\left(t_{i}\right)\right\|_{P}^{2} \leq W\left(\boldsymbol{x}^{-}\left(t_{i}\right)\right)+\beta \leq \frac{1}{2} \delta^{2} \gamma^{2}+\beta$. During the following open time-interval $\left(t_{i}, t_{i+1}\right)$ for which $\boldsymbol{K}(t)=\mathbf{0}$, the function $W$ evolves as $W\left(\boldsymbol{x}^{-}\left(t_{i+1}\right)\right)=$
$W\left(\boldsymbol{x}^{+}\left(t_{i}\right)\right)+\int_{\left(t_{i}, t_{i+1}\right)} \mathrm{d} W$, which may involve impulsive motion due to dissipative impulses $\boldsymbol{\Lambda}$. Let $t_{\mathcal{V}} \in\left(t_{i}, t_{i+1}\right)$ be the time-instance for which $\left\|\boldsymbol{x}^{-}\left(t_{\mathcal{V}}\right)\right\|_{P}=\delta \gamma$. The function $W$ will necessarily decrease during the time-interval $\left(t_{i}, t_{\mathcal{V}}\right)$ due to $(23)$ and $W^{+}-W^{-}=\left(\boldsymbol{x}^{+}\right)^{\mathrm{T}}(\boldsymbol{P} \boldsymbol{D} \boldsymbol{\Lambda})-\frac{1}{2}\|\boldsymbol{D} \boldsymbol{\Lambda}\|_{P}^{2} \leq$ 0 (the state-dependent impulses are passive due to passivity of $\mathcal{H}(\boldsymbol{y})$ ). It therefore holds that

$$
W\left(\boldsymbol{x}^{-}\left(t_{\mathcal{V}}\right)\right) \leq e^{-2\left(1-\frac{1}{\delta}\right) \alpha\left(t_{\mathcal{V}}-t_{i}\right)} W\left(\boldsymbol{x}^{+}\left(t_{i}\right)\right),
$$

because $\mathrm{d} W \leq-2\left(1-\frac{1}{\delta}\right) \alpha W \mathrm{~d} t+\left(W^{+}-W^{-}\right) \mathrm{d} \eta \leq$ $-2\left(1-\frac{1}{\delta}\right) \alpha W \mathrm{~d} t$ for positive measures. Using $W\left(\boldsymbol{x}^{-}\left(t_{\mathcal{V}}\right)\right)=\frac{1}{2} \delta^{2} \gamma^{2}$ and $W\left(\boldsymbol{x}^{+}\left(t_{i}\right)\right) \leq \frac{1}{2} \delta^{2} \gamma^{2}+\beta$ in the exponential decrease (25) gives $\frac{1}{2} \delta^{2} \gamma^{2} \leq$ $e^{-2\left(1-\frac{1}{\delta}\right) \alpha\left(t_{\mathcal{V}}-t_{i}\right)}\left(\frac{1}{2} \delta^{2} \gamma^{2}+\beta\right)$ or $t_{\mathcal{V}}-t_{i} \leq \frac{\delta}{2(\delta-1) \alpha} \ln (1+$ $\left.\frac{2 \beta}{\delta^{2} \gamma^{2}}\right)$. Consequently, if the next impulsive time-instance $t_{i+1}$ of the input is after $t_{\mathcal{V}}$, then the solution $\boldsymbol{x}(t)$ has enough time to reach $\mathcal{V}$. Hence, if the impulsive time-instance of the input are separated by the dwell-time $\tau$ given in (9), i.e. $t_{i+1}-t_{i} \geq \tau$, then the set

$$
\mathcal{W}=\left\{\boldsymbol{x} \in \mathcal{X} \mid \frac{1}{2}\|\boldsymbol{x}\|_{P}^{2} \leq \frac{1}{2} \delta^{2} \gamma^{2}+\beta\right\}
$$

is a compact positively invariant set. Since the size of this positively invariant set is of no concern we can take the limit of the expression for $\tau$ in (9) for $\delta \rightarrow \infty$ : $\lim _{\delta \rightarrow \infty} \frac{\delta}{2(\delta-1) \alpha} \ln \left(1+\frac{2 \beta}{\delta^{2} \gamma^{2}}\right)=0$, which indicates that the dwell-time can be taken arbitrarily small. It therefore suffices to assume that the impulsive inputs $\boldsymbol{K}(t)$ are separated in time (as required in the theorem) to conclude that the system exhibits a compact positively invariant set, defined in (26).

Now, we use Lemma 2 in [19], which formulates that if a dynamic system exhibits a compact positively invariant set, then the existence of a solution that is bounded for $t \in$ $\mathbb{R}$ is guaranteed. We will denote this 'steady-state' solution by $\overline{\boldsymbol{x}}(t)$. The original lemma is formulated for differential equations (possibly with discontinuities, therewith including differential inclusions, with bounded right-hand sides). Here, we use this lemma for measure differential inclusions and would like to note that the proof of the lemma allows for such extensions if we only require continuous dependence on initial conditions. The latter is guaranteed for the Lur'etype measure differential inclusions under study, because the system is exponentially incrementally stable (as shown in the first part of the proof), which implies continuous dependence on initial conditions.

The combination of the fact that all solutions are exponentially stable with the fact that there exists a (steady-state) solution $\overline{\boldsymbol{x}}(t)$, of locally bounded variation, which is bounded for $t \in \mathbb{R}$ for which it is defined, completes the proof of the fact that the system (7) is exponentially convergent according to Definition 4. 